\title{
Do stiffness and asymmetries predict change of direction performance?
}

Sean J. Maloney, Joanna Richards, Daniel G. D. Nixon, Lewis J. Harvey, lain M. Fletcher

Institute of Sports Science and Physical Activity Research, University of Bedfordshire, Bedford, United Kingdom

Corresponding author

Sean Maloney, Research Graduate School, University of Bedfordshire, Polhill Avenue, Bedford, MK41 9EA

Sean.Maloney@beds.ac.uk

+44 7800810099

Keywords

agility, cutting, leg-spring behaviour, spring-mass model, torsional spring model

Acknowledgements

No benefits in any form have been or will be received from any commercial party or grant body related directly or indirectly in relation to this manuscript. The results of the current study do not constitute the endorsement of any product by the authors.

Word Count: 5580 


\section{Do stiffness and asymmetries predict change of direction performance?}

\section{Running head}

Determinants of change of direction speed

Key words

agility, cutting, leg-spring behaviour, spring-mass model, torsional spring model

\section{Acknowledgements}

No benefits in any form have been or will be received from any commercial party or grant body related directly or indirectly in relation to this manuscript. The results of the current study do not constitute the endorsement of any product by the authors. 


\section{Abstract}

Change of direction speed (CODS) underpins performance in a wide range of sports but little is known about how stiffness and asymmetries affect CODS. Eighteen healthy males performed unilateral drop jumps to determine vertical, ankle, knee and hip stiffness, and a CODS test to evaluate left and right leg cutting performance during which ground reaction force data were sampled. A step-wise regression analysis was performed to ascertain the determinants of CODS time. A two-variable regression model explained $63 \%\left(R^{2}=0.63 ; P=0.001\right)$ of CODS performance. The model included the mean vertical stiffness and jump height asymmetry determined during the drop jump. Faster athletes $(\mathrm{n}=9)$ exhibited greater vertical stiffness $(F=12.40 ; P=$ $0.001)$ and less asymmetry in drop jump height $(F=6.02 ; P=0.026)$ than slower athletes ( $n=9$ ); effect sizes were both 'large' in magnitude. Results suggest that overall vertical stiffness and drop jump height asymmetry are the strongest predictors of CODS in a healthy, non-athletic population. 


\section{Introduction}

The ability to quickly and effectively change direction underpins performance in a wide range of sports. For example, change of direction speed (CODS) has been linked to performance in sports such as badminton (Sturgess \& Newton, 2008), soccer (Reilly, Williams, Nevill, \& Franks, 2000), field hockey (Keogh, Weber, \& Dalton, 2003), rugby league (Meir, Newton, Curtis, Fardell, \& Butler, 2001) and basketball (McGill, Andersen, \& Horne, 2012). Understanding the potential determinants of CODS provide the athlete and coach with important information which may better inform the training process.

Young, James, and Montgomery (2002) outline strength, power and reactive strength as the physical factors which may underpin CODS. Reactive strength (a function of the flight time or jump height divided by ground contact time recorded during a drop jump) demonstrated the strongest relationship with CODS test time $(R=-0.54 ; P<$ 0.05). Similar relationships have been also observed by Young, Miller, and Talpey (2015) $(R=-0.65 ; P=0.001)$ and by Delaney et al. $(2015)$ in both dominant $(R=-$ $0.44 ; P<0.05)$ and non-dominant limbs $(R=-0.45 ; P<0.05)$. Reactive strength is a quality which may be closely linked to stiffness; a stiffer leg-spring should facilitate a more rapid release of elastic energy under circumstances were minimal joint or centre of mass displacement is desired, such as during a drop jump or change of direction (Bret, Rahmani, Dufour, Messonnier, \& Lacour, 2002). Indeed, Arampatzis, Schade, Walsh, and Brüggemann (2001) note that higher vertical stiffness is associated with shorter ground contact times during drop jumping.

To the authors' knowledge, only one investigation has sought to examine the effects of stiffness on CODS. Pruyn, Watsford, and Murphy (2014) observed no significant 
relationship between vertical stiffness and 5-0-5 CODS test (examining a single $180^{\circ}$ change of direction from a $15 \mathrm{~m}$ linear acceleration) performance $(R=0.05)$, although they did report significant relationships between performance and stiffness of the musculature surrounding the ankle (medial gastrocnemius: $R=-0.53$, soleus: $R=-$ 0.47 ; both $P<0.05)$. It is important to consider the homogeneity of population sampled by Pruyn et al. (2014); all 18 participants were trained netball players (15.4 \pm 3.0 years of training experience) and exhibited minimal variance in 5-0-5 performance $(2.72 \pm$ $0.18 \mathrm{sec}$ ). The potential relationship between stiffness and CODS would need to be examined in different, possibly less homogenous, populations before conclusions may be drawn. In addition, Pruyn et al. (2014) determined vertical stiffness during a cyclic, unilateral hopping task. It has since been observed that the expression of vertical stiffness and associated asymmetries is highly task dependant (Maloney, Fletcher, \& Richards, 2015). As a change of direction may be characterised as acyclic, ballistic and unilateral in nature, the unilateral drop jump is likely to demonstrate the greatest correspondence to CODS and may therefore carry greater validity as an assessment for vertical stiffness

Several investigations have reported that asymmetries in force/power qualities may be detrimental to athletic performance (Bailey, Sato, Alexander, Chiang, \& Stone, 2013; Bailey, Sato, Burnett, \& Stone, 2015; Bazyler, Bailey, Chiang, Sato, \& Stone, 2014; Bell, Sanfilippo, Binkley, \& Heiderscheit, 2014; Hart, Nimphius, Spiteri, \& Newton, 2014), however, this relationship is not clear in regards to CODS. Whilst eccentric strength asymmetry has been linked to impaired CODS in some investigations (Chaouachi et al., 2012; Lockie, Shultz, Jeffriess, \& Callaghan, 2012), Lockie et al. (2014) reported that athletes with 'typical' asymmetries in unilateral jump 
performance (vertical jump: 10\%; horizontal jump: 3\%; lateral jump: 5\%) did not experience speed detriments.

Asymmetries in COD performance when pushing off the dominant versus nondominant limb have been reported in several investigations (Hart, Lockie, \& Spiteri, 2014; Henry, Dawson, Lay, \& Young, 2013; Young et al., 2002). For example, Hart, Lockie, et al. (2014) reported that Australian footballers demonstrated a performance deficit of $5-10 \%$ between limbs $(\sim 0.72$ seconds; $P \leq 0.001)$ with all players tested exhibiting a directional preference. Given the deterministic model proposed by Young et al. (2002), such asymmetry could be a consequence of an asymmtery in physical qualities. Indeed, Young et al. (2002) noted that athletes who displayed a lateral dominance in COD were likely to have a reactive strength dominance in the limb responsible for the push-off action. Such a relationship is supported by an investigation conducted by Henry et al. (2013) that reported asymmetries in reactive agility performance (discounting decision making time: $5.6 \% ; P=0.04$ ) to mirror asymmetries in reactive strength index $(4.4 \% ; P=0.03)$, although a direct correlation was not reported. Whether asymmetries in dominant versus non-dominant CODS are similarly detrimental to overall CODS performance has not been investigated. Whilst it may seem reasonable to hypothesise that asymmetries in CODS and/or stiffness parameters would be detrimental to overall CODS performance given the current body of evidence, such propositions need to be examined directly.

Ankle stiffness contributes strongly to summative leg stiffness during tasks where minimal joint or centre of mass displacement is desired, for example, during cyclic bilateral hopping (Farley, Houdijk, Van Strien, \& Louie, 1998; Farley \& Morgenroth, 1999; Kim et al., 2013; Kuitunen, Ogiso, \& Komi, 2011) and drop jumping (Arampatzis et al., 2001). Given the findings of Pruyn et al. (2014) and Marshall et al. (2014) this 
relationship appears also to hold true for CODS and, as such, stiffness and asymmetries surrounding the ankle joint may be of particular relevance to CODS. Sugiyama et al. (2014) has previously reported that asymmetries in jump performance were positively correlated with symmetry indices for angular velocity $(R=0.41$; $<$ $0.05)$ and various parameters of angular displacement $(R=0.41-0.52 ; P<0.05)$ of the ankle. These findings suggest that asymmetries in ankle stiffness may negatively influence performance outcomes and further underline the importance of ankle kinematics during performance tasks which require an effective contribution from the stretch shortening cycle.

In summary, variables pertaining to musculoskeletal stiffness have been linked to CODS. Force-related asymmetries have been linked to impaired performance outcomes, but this has not been evaluated in regards to CODS. The aim of the current study was to ascertain the determinants of CODS, with a particular reference to stiffness parameters and asymmetry. It was hypothesised that a) faster performers in the CODS test would display greater vertical stiffness and ankle stiffness, and b) faster performers would display less asymmetry in these variables. 


\section{Method}

\section{Participants}

Eighteen healthy males (age: $22 \pm 4$ years; height: $1.80 \pm 0.08 \mathrm{~m}$; body mass: $81.7 \pm$ $14.9 \mathrm{~kg}$ ) volunteered to participate in the study. A minimum sample size of eighteen participants was determined from an a priori power analysis (G*Power 3.1, HeinrichHeine-Universität, Düsseldorf, Germany) based upon an estimated squared multiple correlation of 0.45 and a power of 0.8 (Beck, 2013). Participants were recreationally active (undertaking $\geq 2.5$ hours of physical activity per week), reported no previous (within the last 12 months) or present lower limb injury and provided informed consent to participate in the study. Full ethical approval was granted by the relevant institutional review board]. All procedures were conducted in accordance with the Declaration of Helsinki.

\section{Experimental trials}

A familiarisation session - a complete simulation of the experimental trial outlined below - was performed seven days prior to the experimental trial. Pilot testing within the same experimental cohort had indicated that a single familiarisation session was appropriate for unilateral drop jumping and for the CODS test. Differences in vertical stiffness during the drop jump were observed in some individuals between an initial testing session and three subsequent sessions, this difference was not apparent for the second session. For the CODS test, individuals did not consistently and accurately contact the force plate during an initial session but did so in subsequent sessions.

All trials were conducted at the same time of day (10:00 - 12:00) for each participant, to alleviate the effects of circadian rhythms. The testing laboratory was controlled at 
an ambient temperature of $25^{\circ} \mathrm{C}$. Participants were instructed to prepare for testing as they would for training. The execution of each experimental trial was monitored by a United Kingdom Strength and Conditioning Association accredited strength and conditioning coach to ensure for consistency of technique.

\section{Warm-up}

All participants completed the same warm-up procedure outlined in Table 1 . The warm-up procedure consisted of 15 dynamic exercises progressing from low to high intensities and from generic to specific movement patterns.

\section{*** Table 1 Here ${ }^{* \star *}$}

A rest period of 60 seconds was prescribed between each of the exercises from the specific movement preparation phase of the warm-up; all other exercises were not prescribed with rest periods. A rest period of 180 seconds was prescribed between the termination of the warm-up and commencement of the testing protocol.

\section{Drop jump testing}

All drop jump assessments were performed on a force plate system (Kistler 9281, Kistler Instruments, Winterthur, Switzerland). The force plate measured $0.6 \mathrm{~m} \times 0.4 \mathrm{~m}$ and was set flush into the laboratory floor as per manufacturer guidelines. Kinetic data were sampled at $1000 \mathrm{~Hz}$ and saved with the use of the manufacturer supplied software (BioWare 3.24, Kistler, Winterthur, Switzerland) for later analysis.

Participants performed a total of three, unshod unilateral drop jumps for each limb; the order in which participants performed unilateral drop jumps was counterbalanced. For the execution of the drop jumps, participants stepped off a $0.18 \mathrm{~m}$ box and performed a vertical jump immediately upon landing. Jumps were performed with participants' 
hands placed behind their head in order not to obscure the reflective markers and to limit the involvement of the upper body during the jump. Participants were instructed to: a) step, not jump, off the box, and b) minimise ground contact time during the landing phase; the execution of every jump was monitored for consistency of technique. Each repetition of the drop jump was separated by 60 seconds to facilitate recovery between efforts (Read \& Cisar, 2001).

\section{Kinematic analysis}

Drop jumping trials were recorded in the sagittal plane using a high-speed video camera (Quintic High-Speed LIVE USB 2, Quintic Consultancy Ltd., Coventry, United Kingdom) recording at $100 \mathrm{~Hz}$. Relative to the force plate, the camera was orientated perpendicular to the anterior-posterior axis, centralised and positioned at a distance of $3.3 \mathrm{~m}$. The camera was mounted on a tripod and set at the height of the participants' knee marker when standing on the box. Reflective joint markers were placed on the distal head of the fifth metatarsal bone (toe), distal aspect of the lateral malleolus (ankle), lateral collateral ligament of the knee at the tibiofemoral gap (knee), greater trochanter (hip) and anterolateral point of $11^{\text {th }}$ rib (torso) on both the left and right sides of the body. Calibration of the video recording was performed prior to the analysis of each trial; the distance between the ankle and hip marker on each leg was used as a scaling reference. Unilateral drop jumps on the left limb were recorded with the participants' left side of the body facing the camera; unilateral drop jumps on the right limb were recorded with the participants' right side of the body facing the camera. Video recordings were automatically digitised using manufacturer provided software (Quintic Biomechanics v21, Quintic Consultancy Ltd., Coventry, United Kingdom). Kinematic data were filtered using a Butterworth fourth-order zero-lag filter (cut-off frequency $20 \mathrm{~Hz}$ ). 
Kinetic analysis

Instants of initial foot contact, take-off and landing were identified from the vertical ground reaction force of each drop jump trial; this was determined as the time-point at which a clear change in force ( $\geq 10 \mathrm{~N}$ from zero) was observed (Lloyd, Oliver, Hughes, \& Williams, 2009). Inverse dynamics was used to express acceleration, velocity and negative displacement of the centre of mass; this was determined from the vertical force trace using the equations described by Blazevich (2007). The vertical velocity of the hip joint marker at the instant of ground contact was used as the initial value for integration.

Net muscle moments were determined using a rigid linked segment model, anthropomorphic data, and an inverse dynamics analysis using the procedures outlined in Winter (2009); the linked segment model was created using Dempster's body segment parameter data (Dempster, 1955).

\section{Stiffness}

Vertical stiffness was calculated as the ratio of peak vertical ground reaction force $(\mathrm{N})$ relative to the peak negative displacement of the centre of mass displacement $(\mathrm{m})$ during the initial ground contact phase (Farley et al., 1998; Farley \& Morgenroth, 1999); this was averaged over the three recorded drop jumps. As vertical stiffness is affected by body size, vertical stiffness values were reported relative to body mass (Farley, Glasheen, \& McMahon, 1993).

Torsional stiffness of the ankle, knee and hip joints were calculated as the ratio of the change in net muscle moment (N.m) to joint angular displacement (rad) between the initial ground contact phase and instant of peak angular displacement (Farley et al., 
1998; Farley \& Morgenroth, 1999); these were averaged over the three recorded drop jumps. Pilot testing indicated that the timing of peak vertical ground reaction forces occurred at the instant of peak joint moments and maximum joint flexions as previously observed by Kuitunen et al. (2011).

\section{Drop jump performance outcomes}

Jump height was determined using the flight-time method as outlined by Linthorne (2001). Reactive strength index was determined as the ratio of flight time to ground contact time (Newton \& Dugan, 2002). Time to peak force was determined as the time difference between the identified instant of initial foot contact and the instant of peak vertical ground reaction force. Overall performance outcomes in the drop jump (vertical stiffness, joint stiffness, jump height, reactive strength index and time to peak force) were obtained by averaging values for the left and right limbs.

\section{Change of direction speed testing}

CODS was assessed using a double cut task highlighted in Figure 1 and performed in a shod condition. Participants were required to perform two $90^{\circ}$ cuts in the same direction (clockwise or anti-clockwise) during each trial and were instructed to complete the task as quickly as possible. Performance time was recorded using two sets of timing gates (TC-Timing System, Brower Timings, Utah, USA) (one set to start the clock, one set to stop the clock) set at the height of the participants' anterior superior iliac spine. Participants performed four consecutive trials in one direction before performing four trials in the other direction; the order in which directions were tested was randomised and counterbalanced. Participants' fastest trial in each direction was subsequently analysed. Overall CODS performance was the sum of 
participants' fastest trials in both directions (best clockwise time + best anticlockwise time). Trials were separated by a recovery duration of 90 seconds.

\section{${ }^{\star \star \star}$ Figure 1 Near Here ${ }^{\star \star \star}$}

To obtain ground reaction force data during the CODS test, the first cut was performed with the push off (outside) foot contacting entirely within the force plate. Trials were excluded if the participant landed outside the confines of the force plate, this was retrospectively checked using video analysis. Considering all trials, a total of seven were excluded, none of which were a participant's fastest trial.

\section{Reliability}

The inter-session coefficient of variation for vertical stiffness was established in a pilot investigation using the same experimental cohort $(n=12)$ over three trials, following a single familiarisation session, a value of $8 \%$ was recorded. The intra-session coefficients of variation for vertical stiffness in the current study were $8 \%$ and $7 \%$ for the stiff and compliant limbs respectively. Intra-session coefficients of variation for joint stiffness were (listed respectively for the stiff and compliant limbs): $2 \%$ and $2 \%$ for the ankle, $3 \%$ and $5 \%$ for the knee and $4 \%$ and $5 \%$ for the hip. Also listed respectively for the stiff and compliant limbs, intra-session coefficients of variation for drop jump height were $6 \%$ and $6 \%$, and for reactive strength index were $4 \%$ and $5 \%$.

Pilot testing $(n=7)$ indicated that the inter-session coefficient of variation (three sessions) for fastest overall CODS test time was 1.1\% (SEM: $0.04 \mathrm{sec}$; ICC: 0.97). The intra-session coefficient of variations for the CODS test in the current study were 1.9\% (SEM: $0.05 \mathrm{sec}$, ICC: 0.95) and 1.9\% (SEM: $0.05 \mathrm{sec;} \mathrm{ICC:} 0.95)$ for the clockwise and anti-clockwise directions respectively. 


\section{Statistical analysis}

For the presentation of results, independent variables were grouped into five categories (CODS test variables, drop jump variables and anthropometric variables) a total of 36 variables. The dependant variable was overall CODS performance time.

Asymmetries were quantified using the symmetry angle, calculated using the procedures outlined by Zifchock et al. (2008). As symmetry angle values may be negative or positive to reflect left or right side dominance, negative values were transformed to positive values prior to statistical analysis in order to evaluate differences solely in the magnitude of asymmetry.

Shapiro-Wilks tests were performed to assess for normality; all variables were considered to be normally distributed given an alpha level of $P>0.05$. The correlation between each variable and overall CODS time was examined using Pearson's $R$. A forward step-wise regression analysis was performed for overall CODS performance using all independent variables. An analysis of standard residuals was carried out, which showed that the data contained no outliers (std. residual min: -1.53 , std. residual max: 1.79). Tests to see if the data met the assumption of collinearity indicated that multicollinearity was not a concern (minimum tolerance: 0.86 , maximum VIF: 1.16). The data met the assumption of independent errors (Durbin-Watson value: 1.44).

For further analysis, performers were median-split into 'fast' $(n=9)$ and 'slow' $(n=9)$ groups based upon overall CODS time. One way analysis of variance (ANOVA) tests were performed to analyse differences between fast and slow groups. Associated pairwise effect sizes (d) (Cohen, 1998) were also calculated and interpreted using the thresholds defined by Hopkins (2003) where: $<0.20=$ trivial, $0.20-0.59=$ small, $0.60-$ $1.19=$ moderate, $1.20-1.99=$ large, and $\geq 2=$ very large. Statistical significance for all 
analyses was set at an alpha level of $P \leq 0.05$ and all statistical procedures were conducted using the Statistical Package for the Social Sciences for Windows (v19.0; SPSS Inc., Chicago, USA).

\section{Results}

Regression analysis

A two-variable regression model explained $63 \%\left(R^{2}=0.629 ; \mathrm{P}=0.001\right)$ of CODS test performance (Table 2). CODS time was predicted by vertical stiffness in the drop jump $($ Beta $=-0.561 ; \mathrm{P}=0.005)$ and by drop jump height asymmetry $($ Beta $=0.391 ; \mathrm{P}<$ 0.035).

\section{${ }^{\star \star \star}$ Table 2 Near Here ${ }^{\star \star *}$}

\section{CODS test variables}

Performance times in the CODS test were significantly different between fast and slow groups $(F=32.02 ; P<0.001)$ and associated with a 'very large' effect size $(d=-2.86)$ (Table 3). The faster group also displayed shorter ground contact times during the test $(F=6.98 ; P=0.018)$, this was associated with a 'large' effect size $(d=-1.33)$. Ground contact time correlated significantly with performance time $(R=0.50 ; P=0.036)$. In regards to force application, asymmetry in summative ground reaction force correlated with performance time $(R=0.470 ; P=0.049)$, but between-group differences were not significant and the effect size was 'small' $(d=-0.23$; $P=0.661)$. A 'moderate' between- 
group effect size $(d=-0.92)$ was observed for asymmetry in medio-lateral ground reaction force, although differences were not significant $(P=0.117)$ and did not correlate to performance time $(R=0.347 ; P=0.159)$.

\section{***Table 3 Near Here ${ }^{\star \star *}$}

\section{Drop jump variables}

Drop jump height was negatively correlated with CODS test time $(R=0.71 ; P=0.001)$. Drop jump height asymmetry was positively correlated with CODS test time $(R=0.60$; $P=0.009)$. The faster group demonstrated greater vertical stiffness $(F=12.40 ; P=$ 0.003) and less asymmetry in jump height $(F=6.02 ; P=0.026)$ during the drop jump (Table 4); these effect sizes were both 'large' and 'moderate' (vertical stiffness: $d=$ 1.76, jump height asymmetry: $d=-1.28)$. Effect size analyses also revealed a 'moderate' differences in ankle stiffness $(d=0.62 ; P=0.280)$ and vertical ground reaction force relative to body mass $(d=0.79 ; P=0.149)$, although these differences were not significant at the applied level.

\section{${ }^{\star \star *}$ Table 4 Near Here ${ }^{\star \star *}$}

The direction of asymmetry for CODS test performance did not appear to correspond to the direction of either jump height (Figure 2) or vertical stiffness (Figure 3) asymmetry.

\section{${ }^{* * *}$ Figures 2 \& 3 Near Here***}

\section{Anthropometric variables}

Performers in the fast group were significantly shorter in stature than those in the slow group $(F=4.77 ; P=0.044)$ and tended to have shorter lower limbs $(F=3.07 ; P=$ 
0.099) (Table 5); both effect sizes were 'moderate' (height: $d=-1.14$, leg length: $d=-$ 0.89). The correlation between height and performance time approached significance $(R=0.44 ; P=0.065)$. 


\section{Discussion}

The current study sought to ascertain the determinants of COD performance with a particular reference to stiffness parameters and asymmetry. It was hypothesised that a) vertical stiffness and ankle stiffness would be greater in faster performers, and b) faster performers will display less asymmetry for these variables. Mean vertical stiffness and asymmetry in jump height, both determined during a single leg drop jump test, were the strongest predictors of the time taken to complete the CODS test employed in the current study. As such, the first hypothesis holds only partially true as ankle stiffness was not a strong predictor of performance. The second hypothesis is rejected as asymmetries in vertical stiffness and ankle stiffness did not predict performance.

Vertical stiffness was the strongest predictor of CODS according to the regression model, greater vertical stiffness led to quicker performance times. Between-group analyses also revealed a 'large' and significant difference between fast and slow groups. Pruyn et al. (2014) had previously examined the potential relationship between stiffness and CODS, although employed a unilateral hopping task which may not represent the acyclic, ballistic nature of CODS tasks. Contrary to the results of the current study, Pruyn et al. (2014) reported that vertical stiffness was not correlated to CODS and that performance times of median-split stiff and compliant groups were not different. The reduced homogeneity of the population sample in the current study in comparison to that of Pruyn et al. (2014) (highly trained female netballers) may explain this discordance in results. Intuitively, it would seem likely that athletes exhibiting greater vertical stiffness during the drop jump would exhibit great leg stiffness during a change of direction. Indeed, the current study reports that faster athletes displayed shorter ground contact times than slower performers, in line with the results of previous 
investigations (Marshall et al., 2014; Sasaki, Nagano, Kaneko, Takakuni, \& Fukubayashi, 2011), and is likely to be indicative of greater leg stiffness during the change of direction. Future research should seek to directly examine leg stiffness during changes of direction in order to better explore the relationship between stiffness and CODS, the absence of a three-dimensional motion capture system precluded such measurements to be used in the current study.

Regression analyses revealed that asymmetry in single leg drop jump height was the second strongest predictor of CODS performance time such that lesser asymmetries were associated with quicker times. Between-group analyses also indicated a 'large' and significant difference between median-split fast and slow performers. Whilst previous investigations have associated asymmetries in force-related parameters with impaired athletic performance (Bailey et al., 2013; Bailey et al., 2015; Bazyler et al., 2014; Bell et al., 2014; Hart, Nimphius, et al., 2014), the relationship between asymmetry and CODS is not clear. Two investigations have noted reductions in CODS performance where an eccentric strength asymmetry is apparent (Chaouachi et al., 2012; Lockie et al., 2012). Eccentric strength is likely to underpin an athlete's ability to effectively utilise the stretch shortening cycle during movements such as drop jump or a change of direction where minimal joint displacement may be desired (Cormie, McGuigan, \& Newton, 2010). It is therefore possible that eccentric strength asymmetries could underpin asymmetries in jump height or CODS, although such propositions would need to be examined directly.

Lockie et al. (2014) examined the relationship between multi-planar unilateral jumping performance and CODS in well-trained, multidirectional team-sport athletes. Lockie et al. (2014) noted asymmetries of $10.4 \%( \pm 10.8 \%), 3.3 \%( \pm 3.0 \%)$ and $5.1 \%( \pm 3.9 \%)$ in vertical, horizontal and lateral jump performance respectively, but reported that these 
asymmetries were not related to 5-0-5 or T-test performance. Previously, Hoffman, Ratamess, Klatt, Faigenbaum, and Kang (2007) had also reported that asymmetries in unilateral vertical jump power of $9.7 \%( \pm 6.9 \%)$ were not associated with reductions in 3-cone drill performance in collegiate American footballers. In neither of these studies do the investigator's data permit the calculation of a symmetry angle. The average jump height asymmetry in the current study was $13 \%( \pm 12 \%)$ when expressed as a symmetry index as in Lockie et al. (2014) and Hoffman et al. (2007), equating to a symmetry angle of $4.3 \%$. The larger asymmetries present in the current population could potentially explain why CODS impairments were observed.

It is also possible that the athletic background of participants could explain why asymmetry was detrimental to CODS in this instance. Lockie et al. (2014) hypothesised that more skilled performers may be better able to initiate technical adjustments in response to strength or power asymmetries than recreationally trained participants such as those sampled in the current study. However, in the current study the direction of asymmetries in drop jump variables did not correspond well with the direction of asymmetry in the CODS test. It is therefore conceivable that the observed association between asymmetry and CODS performance is purely indicative of participants' current athletic ability or training status. Indeed, Bazyler et al. (2014) reported that asymmetries are likely to be greater in weaker individuals. Across a seven week bilateral training programme, Bazyler et al. (2014) also noted that as strength increased there was a concomitant decrease in asymmetry in weaker individuals. Future investigations should seek to determine whether asymmetries in the variables highlighted in the current study are associated with CODS in an athletic population. 
In addition to the asymmetries in jump height observed during the drop jump test, the results of the current study suggest that asymmetries in the application of force during CODS test may also be linked to performance time. Most notably, asymmetries in summative ground reaction force were linked to impaired performance. Condello, Kernozek, Tessitore, and Foster (2015) similarly reported between-limb differences in ground reaction forces, although reported no difference in ground contact time (no overall 'performance' time was recorded). If greater forces, relative to body mass, can be applied to the ground without negatively affecting ground contact time then this is likely to be beneficial to CODS performance due to the necessary impulse required to change direction being generated quicker. Between-group differences in the current study also suggested that slower performers exhibited greater asymmetry in mediolateral ground reaction force, however, this variable did not directly correlate to performance time. The amount of force expressed in the medio-lateral direction in particular (i.e. the direction of intended travel) is most likely to result in improved CODS performance (Shimokochi, Ide, Kokubu, \& Nakaoji, 2013).

Reactive strength index is a quality purported to be closely linked to vertical stiffness (Arampatzis et al., 2001; Bret et al., 2002). Whilst previous investigations had reported significant correlations $(R=-0.44--0.65)$ between reactive strength index and CODS performance (Delaney et al., 2015; Young et al., 2002; Young et al., 2015), the current study did not observe this relationship to be significant. Although reactive strength index and leg stiffness may be closely linked, that these terms are not synonymous and should not be used interchangeably.

Vertical stiffness is a function of vertical ground reaction force and centre of mass displacement (Farley et al., 1998; Farley \& Morgenroth, 1999), therefore asymmetries in either of these variables could influence asymmetries in vertical stiffness. Despite 
the magnitude of the difference in vertical stiffness between the fast and slow groups, differences in vertical ground reaction force and centre of mass displacement were not significant and only the former was associated with a 'moderate' effect size. This is not in agreement with a previous findings which identify centre of mass displacement as a strong factor in determining vertical stiffness asymmetry (Maloney et al., 2015), although it should be noted that the current study expressed vertical ground reaction force relative to body mass whereas the previous investigation analysed raw values. Nonetheless, it is likely that asymmetries in both factors have an additive effect that is exhibited in vertical stiffness asymmetry (Maloney et al., 2015).

Centre of mass displacement is deemed to be a global representation of how the legspring deforms in response to ground reaction force (Butler, Crowell III, \& Davis, 2003), although does not consider the respective contribution of individual joints or 'springs' (Pearson \& McMahon, 2012). Within the leg-spring, the ankle is the least stiff joint and has been shown to regulate vertical stiffness in bilateral hopping (Farley et al., 1998; Farley \& Morgenroth, 1999; Kim et al., 2013; Kuitunen et al., 2011) and drop jumping (Arampatzis et al., 2001). Conversely, adjustments in knee stiffness appear important in optimising torque output rather than in the modulation of vertical stiffness (Kuitunen et al., 2011); the anatomy of the knee extensors in relation to the plantar flexors facilitates greater moments at the knee versus the ankle (Alexander \& Ker, 1990). Comparisons between the fast and slow groups (Table 4) suggest a potential reliance on different movement strategies during the drop jump; the fast group exhibit 'moderately' greater ankle stiffness but lower values for knee and hip stiffness. These differences in ankle stiffness may ultimately explain the differences in CODS performance. Given the low intra-session coefficients of variation for joint stiffness 
reported in the current study (ankle: $2 \%$, knee and hip: $\leq 5 \%$ ), it is likely that such differences are not a consequence of intra-limb variability.

In the investigation by Pruyn et al. (2014), stiffness of the medial gastrocnemius and soleus, determined by quasi-isometric myometry, was shown to differentiate fast and slower performers where vertical stiffness did not. Marshall et al. (2014) also observed a significant correlation plantar flexor moment at the ankle $(R=-0.65)$ and ankle power $(R=-0.77)$ with the time to complete a lateral cutting task. These results suggest that stiffness around the ankle may contribute to CODS although this was not examined directly in either investigation. The current study reports moderate between-group differences in both ankle stiffness and ankle stiffness asymmetry such that faster performers had stiffer ankles and displayed less asymmetry. However, this relationship was not statistically robust and correlations with CODS performance time were not observed. Whilst ankle stiffness is no doubt important during a change of direction, particularly given its likely governance of vertical stiffness (Arampatzis et al., 2001; Farley et al., 1998; Farley \& Morgenroth, 1999; Kim et al., 2013; Kuitunen et al., 2011), it appears that this is not an important determinant of CODS performance in its own right, at least when evaluated in a drop jump task. The current study suggests that summative stiffness of the leg may be more important to CODS than the stiffness of any individual joint, but future investigations should seek to examine ankle stiffness during the CODS task directly. Moreover, a three-dimensional motion analysis of the CODS test would demonstrate how additional kinematic factors (i.e. pelvic lateral tilt and thorax rotation (Marshall et al., 2014)) influence and interact with stiffness variables.

In conclusion, the mean vertical stiffness and jump height asymmetry determined during a single leg drop jump were the strongest predictors of CODS in recreationally 
trained males. The single leg drop jump test may provide coaches and practitioners with a tool to not only assess an individual's stiffness profile, but also to quantify specific factors linked to CODS. However, the efficacy of this tool should first be evaluated in athletic populations. This assessment may be used to inform the training process and evaluate the impact of a specific exercise intervention. Further research is required to determine if the modulation of these factors through training may improve CODS. 


\section{References}

Alexander, R. M., \& Ker, R. F. (1990). The architecture of leg muscles. In J. M. Winters \& S.-Y. Woo (Eds.), Multiple muscle systems: biomechanics and movement organization (pp. 568-577). New York: Springer-Verlag.

Arampatzis, A., Schade, F., Walsh, M., \& Brüggemann, G. P. (2001). Influence of leg stiffness and its effect on myodynamic jumping performance. Journal of Electromyography and Kinesiology, 11(5), 355-364

Bailey, C., Sato, K., Alexander, R., Chiang, C.-Y., \& Stone, M. H. (2013). Isometric force production symmetry and jumping performance in collegiate athletes. Journal of Trainology, 2, 1-5

Bailey, C. A., Sato, K., Burnett, A., \& Stone, M. H. (2015). Force production asymmetry in male and female athletes of differing strength levels. International Journal of Sports Physiology and Performance, 10(4), 504-508

Bazyler, C. D., Bailey, C. A., Chiang, C.-Y., Sato, K., \& Stone, M. H. (2014). The effects of strength training on isometric force production symmetry in recreationally trained males. Journal of Trainology, 3(1), 6-10

Beck, T. W. (2013). The importance of a priori sample size estimation in strength and conditioning research. Journal of Strength and Conditioning Research, 27(8), 2323-2337

Bell, D. R., Sanfilippo, J. L., Binkley, N., \& Heiderscheit, B. C. (2014). Lean mass asymmetry influences force and power asymmetry during jumping in collegiate athletes. Journal of Strength and Conditioning Research, 28(4), 884-891

Blazevich, A. J. (2007). Position, Velocity and Acceleration. In A. J. Blazevich (Ed.), Sports Biomechanics: The Basics: Optimising Human Performance (pp. 1-14). London, England: A \& C Black Publishers Ltd.

Bret, C., Rahmani, A., Dufour, A. B., Messonnier, L., \& Lacour, J. R. (2002). Leg strength and stiffness as ability factors in $100 \mathrm{~m}$ sprint running. Journal of Sports Medicine and Physical Fitness, 42(3), 274-281

Butler, R. J., Crowell III, H. P., \& Davis, I. M. (2003). Lower extremity stiffness: implications for performance and injury. Clinical Biomechanics, 18(6), 511-517

Chaouachi, A., Manzi, V., Chaalali, A., Wong, D. P., Chamari, K., \& Castagna, C. (2012). Determinants analysis of change-of-direction ability in elite soccer players. Journal of Strength and Conditioning Research, 26(10), 2667-2676

Cohen, J. (1998). Statistical power analysis for the behavioral sciences (2nd ed.). New Jersey: Lawrence Erlbaum.

Condello, G., Kernozek, T. W., Tessitore, A., \& Foster, C. (2015). Biomechanical analysis of a change of direction task in collegiate soccer players. International Journal of Sports Physiology and Performance. doi: 10.1123/ijspp.2014-0458

Cormie, P., McGuigan, M. R., \& Newton, R. U. (2010). Changes in the eccentric phase contribute to improved stretch-shorten cycle performance after training. Medicine \& Science in Sport \& Exercise, 42(9), 1731-1744

Delaney, J. A., Scott, T. J., Ballard, D. A., Duthie, G. M., Hickmans, J. A., Lockie, R. G., \& Dascombe, B. J. (2015). Contributing factors to change-of-direction ability in professional rugby league players. Journal of Strength and Conditioning Research. doi: 10.1519/JSC.0000000000000960

Dempster, W. T. (1955). Space requirements of the seated operator. Ohio: WrightPatterson Air Force Base. 
Farley, C. T., Glasheen, J., \& McMahon, T. A. (1993). Running springs: speed and animal size. The Journal of Experimental Biology, 185, 71-86

Farley, C. T., Houdijk, H. H. P., Van Strien, C., \& Louie, M. (1998). Mechanism of leg stiffness adjustment for hopping on surfaces of different stiffnesses. Journal of Applied Physiology, 85(3), 1044-1055

Farley, C. T., \& Morgenroth, D. C. (1999). Leg stiffness primarily depends on ankle stiffness during human hopping. Journal of Biomechanics, 32(3), 267-273

Hart, N. H., Lockie, R. G., \& Spiteri, T. (2014). Detecting deficits in change of direction performance using the pre-planned, multi-directional AFL agility test. Journal of Strength and Conditioning Research. doi: 10.1519/JSC.0000000000000587

Hart, N. H., Nimphius, S., Spiteri, T., \& Newton, R. U. (2014). Leg strength and lean mass symmetry influences kicking performance in Austrailian football. Journal of Sports Science and Medicine, 13(1), 157-165

Henry, G. J., Dawson, B., Lay, B. S., \& Young, W. B. (2013). Relationships between reactive agility movement time and unilateral vertical, horizontal and lateral jumps. Journal of Strength and Conditioning Research. doi: 10.1519/JSC.0b013e3182a20ebc

Hoffman, J. R., Ratamess, N. A., Klatt, M., Faigenbaum, A. D., \& Kang, J. (2007). Do bilateral power deficits influence direction-specific movement patterns? Research in Sports Medicine, 15(2), 125-132

Hopkins, W. G. (2003). A spreadsheet for analysis of straightforward controlled trials. Sportscience, 7 ,

Keogh, J. W. L., Weber, C. L., \& Dalton, C. T. (2003). Evaluation of anthropometric, physiological, and skill related tests for talent identification in female field hockey. Canadian Journal of Applied Physiology, 28(3), 397-409

Kim, W., João, F., Tan, J., Mota, P., Vleck, V., Aguiar, L., \& Veloso, A. (2013). The natural shock absorption of the leg spring. Journal of Biomechanics, 46(1), 129136

Kuitunen, S., Ogiso, K., \& Komi, P. V. (2011). Leg and joint stiffness in human hopping. Scandinavian Journal of Medicine and Science in Sports, 21(6), e157-e167

Linthorne, N. P. (2001). Analysis of standing vertical jumps using a force platform. American Journal of Physics, 69(11), 1198-1204

Lloyd, R. S., Oliver, J. L., Hughes, M. G., \& Williams, C. A. (2009). Reliability and validity of field-based measures of leg stiffness and reactive strength index in youths. Journal of Sports Sciences, 27(14), 1565-1573

Lockie, R. G., Callaghan, S. J., Berry, S. P., Cooke, E. R., Jordan, C. A., Luczo, T. M., \& Jeffriess, M. D. (2014). Relationship between unilateral jumping ability and asymmetry on multidirectional speed in team-sport athletes. Journal of Strength and Conditioning Research, 28(12), 3557-3566

Lockie, R. G., Shultz, A. B., Jeffriess, M. D., \& Callaghan, S. J. (2012). The relationship between bilateral differences of knee flexor and extensor isokinetic strength and multi-directional speed. Isokinetics and Exercise Science, 20(3), 211-219

Maloney, S. J., Fletcher, I. M., \& Richards, J. (2015). A comparison of methods to determine bilateral asymmetries in vertical leg stiffness. Journal of Sports Sciences. doi: 10.1080/02640414.2015.1075055

Marshall, B. M., Franklyn-Miller, A. D., King, E. A., Moran, K. A., Strike, S. C., \& Falvey, É. C. (2014). Biomechanical factors associated with time to complete a change of direction cutting maneuver. Journal of Strength and Conditioning Research, 28(10), 2845-2851 
McGill, S. M., Andersen, J. T., \& Horne, A. D. (2012). Predicting performance and injury resilience from movement quality and fitness scores in a basketball team over 2 years. Journal of Strength and Conditioning Research, 26(7), 1731-1739

Meir, R., Newton, R., Curtis, E., Fardell, M., \& Butler, B. (2001). Physical fitness qualities of professional rugby league football players: Determination of positional differences. Journal of Strength and Conditioning Research, 15(4), 450-458

Newton, R. U., \& Dugan, E. (2002). Application of strength diagnosis. Strength \& Conditioning Journal, 24(5), 50-59

Pearson, S. J., \& McMahon, J. (2012). Lower limb mechanical properties: determining factors and implications for performance. Sports Medicine, 42(11), 929-940

Pruyn, E. C., Watsford, M., \& Murphy, A. (2014). The relationship between lower-body stiffness and dynamic performance. Applied Physiology, Nutrition, and Metabolism, 39(10), 1144-1150

Reilly, T., Williams, A. M., Nevill, A., \& Franks, A. (2000). A multidisciplinary approach to talent identification in soccer. Journal of Sports Sciences, 18(9), 695-702

Sasaki, S., Nagano, Y., Kaneko, S., Takakuni, S., \& Fukubayashi, T. (2011). The relationship between performance and trunk movement during change of direction. Journal of Sports Science and Medicine, 10(1), 112-118

Shimokochi, Y., Ide, D., Kokubu, M., \& Nakaoji, T. (2013). Relationships among performance of lateral cutting maneuver from lateral sliding and hip extension and abduction motions, ground reaction force, and body center of mass height. Journal of Strength and Conditioning Research, 27(7), 1851-1860

Sturgess, S., \& Newton, R. U. (2008). Design and implementation of a specific strength program for badminton. Strength \& Conditioning Journal, 30(3), 34-41

Sugiyama, T., Kameda, M., Kageyama, M., Kiba, K., Kanehisa, H., \& Maeda, A. (2014). Asymmetry between the dominant and non-dominant legs in the kinematics of the lower extremities during a running single leg jump in collegiate basketball players. Journal of Sports Science and Medicine, 13(951-957)

Winter, D. A. (2009). Kinetics: forces and moments of force Biomechanics and Motor Control of Human Movement (4th ed., pp. 107-138). New Jersey: John Wiley \& Sons, Inc.

Young, W., James, R., \& Montgomery, I. (2002). Is muscle power related to running speed with changes of direction? Journal of Sports Medicine and Physical Fitness, 42(3), 282-288

Young, W. B., Miller, I. R., \& Talpey, S. W. (2015). Physical qualities predict changeof-direction speed but not defensive agility in Australian rules football. Journal of Strength and Conditioning Research, 29(1), 206-212

Zifchock, R. A., Davis, I., Higginson, J., \& Royer, T. (2008). The symmetry angle: A novel, robust method of quantifying asymmetry. Gait \& Posture, 27(4), 622-627 